\title{
ÉTUDE STATISTIQUE
}

A la remise de son rapport annuel sur l'immigration 1982 au Parlement le 2 novembre dernier, le ministre de l'Emploi et de l'Immigration, M. Lloyd Axworthy a rendu public le nombre total de réfugiés que le Canada prévoit accueillir en 1982. Les chiffres prévus pour 1981 et le nombre d'arrivées au Canada à date cette année sont indiqués pour fins de comparaison.

\section{NOMBRE TOTAL DES RÉFUGIÉS}

Europe de l'Est

6000

Indochine

Amérique latine et les Caraibes

Afrique

Moyen Orient
4000

1000

500

400

Nombre total d'arrivées prévu, tenant compte des imprévus et des autres régions du globe

14000

Ces chiffres ne tiennent pas compte des réfugiés parrainés par des groupes privés.

1981 NOMBRE TOTAL DES RÉFUGIÉS

Arrivées prévues

Europe de l'Est

Indochine

Amérique latine et Caraïbes

Afrique

Autres régions du globe

4000 (porté à 5000 )

Arrivées à compter du 1er août

2332

8000

6250

1000

200 (porté à 400)

300

Nombre total d'arrivées prévu, tenant compte des imprévus $\quad \mathbf{1 6 , 0 0 0}$

De plus, le gouvernement estime que 5000 réfugiés parrainés par des groupes privés arriveront au Canada cette année; à date, seulement 1593 ont été admis.

REFUGE est une publication mensuelle autonome à but non lucratif, dont les fonds proviennent de subventions de la Fondation canadienne pour les réfugiés et de Wintario, de dons offerts par des particuliers et des frais d'abonnement facultatifs de $\$ 10$ pour 10 numéros. Si ses articles se prêtent à la discussion, ils ne reflètent pas nécessairement l'opinion de ceux qui accordent des fonds à la revue ni celle de son personnel ou de son comité de rédaction.

\section{Comité de rédaction}

Stuart Beaveridge, Victoria Daniel Ferguson, Toronto Douglas Cohen, Montréal

Claudió Duran, Toronto

Arie van Eek, Burlington
Dawn MacDonald, Toronto

Michael Pitman, Toronto Linda Weigl, Regina
Toutes les nominations seront les bienvenues.

Rédacteur: Kristin Hanson

Adjoint à la recherche pour l'été: Shirra Freeman.

REFUGE is available in both English and French.

Sauf indication contraire et à moins qu'ils ne soient tirés d'une autre publication, tous les textes parus dans REFUGE peuvent être reproduits sans permission. Le nom de l'auteur et de la source, s'ils sont connus devraient cependant être mentionnés.

Nous tenons à remercier chaleureusement les artistes de Dreadnaught Cooperative, Inc de Toronto qui nous ont fait don du logo de REFUGE. 